\title{
Chromatin Dynamics
}

\section{Contents}

2.1 Basic Nuclear Activities - 30

2.2 Connecting Nucleosomes to DNA Sequence - 31

2.3 Nucleosome Remodeling - $\mathbf{3 3}$

2.3.1 A Template for Transcription - 33

2.3.2 Chromatin Remodeling Complexes -37

2.4 Nucleosome Assembly - 42

2.4.1 Histone Variants and Histone Chaperones - 42

2.4.2 The Replication Fork: Still the Major Enigma in Epigenetics - 43

References - 46 


\section{What You Will Learn in This Chapter}

The nucleus of a eukaryotic cell is a very busy place. Not only during replication of the DNA, but at any time in the cell cycle specific enzymes need access to genetic information to process reactions such as transcription and DNA repair. Yet, the nucleosomal structure of chromatin is primarily inhibitory to these processes and needs to be resolved in a highly orchestrated manner to allow developmental, organismal, and cell type-specific nuclear activities. This chapter explains how nucleosomes organize and structure the genome by interacting with specific DNA sequences. Variants of canonical histones can change the stability of the nucleosomal structure and also provide additional epigenetic layers of information. Chromatin remodeling complexes work locally to alter the regular beads-ona-string organization and provide access to transcription and other DNA processing factors. Conversely, factors like histone chaperones and highly precise templating and copying mechanisms are required for the reassembly of nucleosomes and reestablishment of the epigenetic landscape after passage of activities processing DNA sequence information. A very intricate molecular machinery ensures a highly dynamic yet heritable chromatin template.

\subsection{Basic Nuclear Activities}

The eukaryotic genome is packaged into a nucleosomal beads-on-a-string architecture (see book Chap. 1 of Wutz). This allows the long but thin DNA fiber to be protected, condensed, and organized in the cell nucleus. For example, tight packaging of the genome is used actively to suppress deleterious sequences to become mobilized, like transposable or retroviral elements. However, DNA acts as a template for a number of nuclear processes which require access to the genetic information and, thus, an open chromatin conformation. Some of the associated enzymes use, for example, the sequence of a DNA strand to accomplish their work. However, nucleosomal DNA is double-stranded requiring that the DNA fiber be freed from histone packaging before these nuclear activities can be carried out. A number of mechanisms evolved to counter the primarily repressive nature of the nucleosome, permitting flexible and responsive nuclear activities.

A prime example in this respect is the process of transcription. The eukaryotic cell has three RNA polymerases, which are distinguished by the RNA products they produce. Their mode of action is similar, though, requiring the sequence information provided by an open DNA strand to synthesize the complementary RNA molecule (Cramer 2019). Before this process can start, gene activation mechanisms ensure that particular transcription factors (TFs) can access their specific target sequences, further requiring a remodeling of the protective nucleosomal structure at their sites of action, like enhancers. Similarly, during the process of elongation, the RNA polymerase complex encounters a nucleosomal structure, which needs not only to be removed for the synthesis of the transcript but also reassembled in an epigenetically conform manner after passage. All these steps are in conflict with the primarily static interaction of DNA and histones and require a dynamic chromatin structure responding to external signals, enzymatic processing, and mechanical forces.

The process of DNA replication uses the activity of DNA polymerases templating on single-stranded DNA the production of an exact copy of the genetic material (Hammond et al. 2017). As such, sites of origins, where replication starts, have to be 
freed of nucleosomes to allow the factors to bind DNA and assemble the replication machinery. Nucleosomes are removed ahead of the advancing replication fork and reassembled behind the site of replication. In addition, histone modifications, DNA methylation, and other chromatin associated factors are reestablished to maintain the epigenetic landscape. Indeed, at the replication site, two distinct mechanisms of epigenetic inheritance are taking place, of which we do understand one very well. The beauty of the complementary DNA structure revealed immediately how this molecule can self-template its own copy. Conversely, we know much less about how heritable epigenetic information is duplicated and faithfully replaced after passage of the replication fork. While we understand comparatively well the duplication of DNA methylation patterns on newly synthesized DNA (see book $>$ Chap. 1 of Wutz), we still lack a similarly comprehensive understanding of how protein-encoded information, including histone marks and histone variants is maintained in a heritable manner.

Other nuclear processes like DNA repair or recombination require the alignment of DNA sequences and, hence, a local removal of nucleosomal structures. Dedicated processes ensure fast repair of open damaged sites and the search factors required to align complementary genome sections for sequence exchange (PapamichosChronakis and Peterson 2013; Misteli and Soutoglou 2009).

Still other nuclear processes like the addition of telomere sequences to the end of chromosomes, the generation of anchoring points for the segregation of chromosomes, or the silencing of transposable or repeat elements in heterochromatic sequences are dependent on specific chromatin structures for their functional performance. This shows that chromatin needs to be dynamically regulated to support nuclear processes during the cell cycle in a predictable and reliable manner. The dampening nature of nucleosomes has also a positive effect, however. Transcription factors have many more presumptive binding sites in the genome than are observed in vivo. In combination with highly processive enzymes like RNA polymerase II, this can result in spurious and unwanted genome transcription. Such "expression noise" is kept in check by the nucleosomal packaging and only regulated chromatin accessibility will allow the basic nuclear processes to trigger their activities at the specific and required sites in the genome.

\subsection{Connecting Nucleosomes to DNA Sequence}

As packaging entities for an entire genome, it is clear that histones cannot rely on a similar DNA sequence-specificity as transcription factors. Nevertheless, as a nucleosomal unit, histone proteins are intimately interacting with the DNA that bends around the histone core. This can only be achieved through distinctive chemical interactions between the nucleic acid and the histones. As such, nucleosome assembly is thermodynamically preferred for some sequences in comparison to others, suggesting that chromatin architecture is governed by the sequence of the genome (Parmar and Padinhateeri 2020; Hughes and Rando 2014; Struhl and Segal 2013). Certain sequence determinants, like particular dinucleotides, affect DNA bending and, in consequence, nucleosome formation. Optimal nucleosome formation occurs when bendable dinucleotides (AT and TA) occur on the face of the helical repeat (approximately every $10 \mathrm{bp}$ ) that directly interacts with histones (• Fig. 2.1). 
a

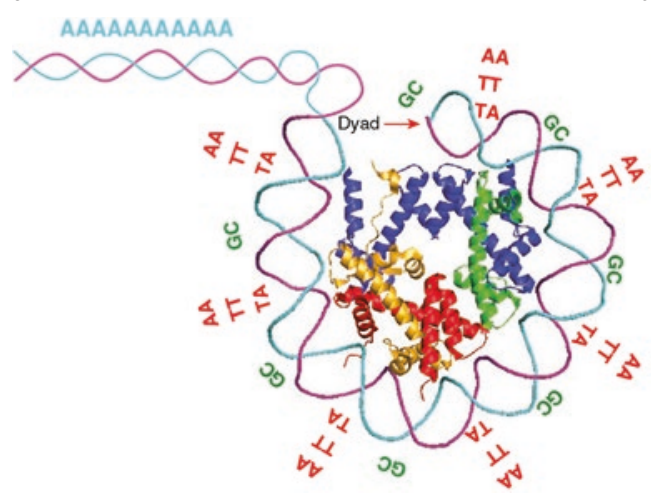

b
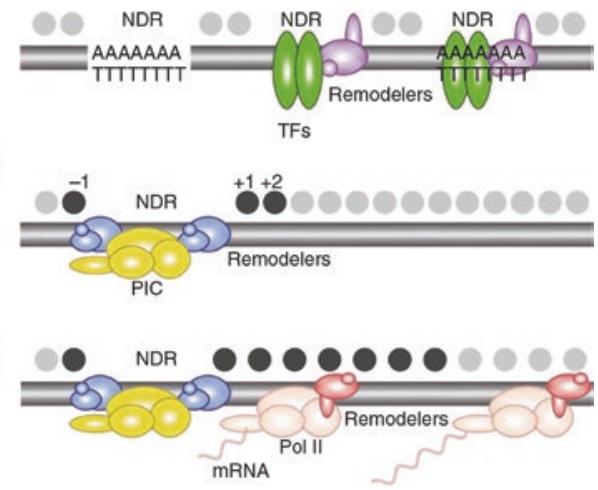

- Fig. 2.1 Sequence determining nucleosomal position. a Nucleosome sequence preferences. Within the $147 \mathrm{bp}$ that are wrapped around the histone octamer, there is a preference for distinctive dinucleotides that recur periodically at the DNA helical repeat and are known to facilitate the sharp bending of DNA around the nucleosome. These include $\sim 10$-bp periodic AA, TT, or TA dinucleotides that oscillate in phase with each other and out of phase with $\sim 10$-bp periodic GC dinucleotides. The linker regions exhibit a strong preference for sequences that resist DNA bending and, thus, disfavor nucleosome formation. Among these, poly $(\mathrm{dA}: \mathrm{dT})$ tracts and their variants are the most dominant and highly enriched in eukaryotic promoters. b Determinants of nucleosome positioning. (top) Nucleosome-depleted regions (NDRs) are generated either by poly(dA:dT) tracts and/or by transcription factors and the nucleosome remodeling complexes they recruit (see below). Gray circles indicate nucleosomes. (middle) Nucleosomes located at highly preferred positions (black circles) flanking the NDR are generated by nucleosome-remodeling complexes (for example, Isw2 and RSC, likely in a transcription-independent manner), and fine-tuned by the Pol II preinitiation complex (PIC) and associated factors. (bottom) Positioning of the more downstream nucleosomes depends on transcriptional elongation as well as the recruitment of nucleosome-remodeling activities (for example, Chd1 and Isw1) and histone chaperones by the elongating Pol II machinery. (From Struhl and Segal (2013))

The exact position of the histone octamer with respect to the 10-bp helical repeat of the DNA double helix is termed 'rotational positioning' and, thus, sequence is a critical determinant for the assembly of the nucleosome. However, protein-DNA interactions are not only recognizing base readout but are also dependent on the local shape structure of the DNA fiber, which is determined by the local sequence of bases (Rohs et al. 2010). Local kinks in the DNA fiber or variable width of the minor groove influence the efficiency of nucleosome build-up. Conversely, some homopolymeric sequences like poly $(\mathrm{dA}: \mathrm{dT})$ and poly $(\mathrm{dG}: \mathrm{dC})$ are rather stiff and are strongly inhibitory to nucleosome formation. Such sequences are often found at positions where nucleosome presence is reduced to allow transcription factors or polymerases access to enhancers or promoters, respectively. Hence, a combination of positively and negatively acting DNA sequences has a strong impact on the positioning of nucleosomes in the genome. Indeed, this information allowed predicting fairly accurately the position of nucleosomes in the yeast genome by computational methods. Similar attempts for more complex genomes have failed, probably because other factors might strongly influence the assembly and positioning of nucleosomes (see below). This indicates that sequence-dependent effects might be less important for nucleosome positioning in higher organisms.

In addition to a local interdependence between DNA sequence and nucleosome position, a number of DNA regulatory elements with a strong impact on large-scale 
chromatin conformations have evolved. Highly repressed chromatin, like heterochromatin, is built by multiprotein complexes with a strong polymerization activity. They package the nucleosomal fiber over an extended chromosomal region into a condensed and regular arrangement (see book $>$ Chap. 1 of Wutz). Heterochromatic regions do not only show a local regular array of condensed nucleosomes but also a clustering in the 3D space of the nucleus. This highly repressive structure can potentially immerse nearby active genes. The propensity of heterochromatin to spread along the chromosome can be observed in position effect variegation (PEV). After chromosomal translocations, repressive heterochromatin can "spread" into regions with euchromatic genes inhibiting their normal regulated activity. Normally, the presence of regulatory elements, termed boundary elements or insulators, restrains this expansive behavior of heterochromatin (Valenzuela and Kamakaka 2006). In cases where these insulators are lost, a stochastic spreading of heterochromatin in neighboring euchromatic genes can be observed, resulting in a variable expression of these genes.

However, also at the local level, different chromatin domains and gene regulatory units need to be appropriately controlled such that they can exert their function not over the linear dimension of the DNA fiber but in the true 3D space of the nucleus. At complex genetic loci, such insulator elements regulate the tissue-specific and developmental-specific interaction of distant enhancer elements with their corresponding promoter region. Additionally, such elements prevent the spreading of repressive chromatin, like PcG-chromatin (see $>$ Chap. 3 of Paro), into neighboring active expression domains. Technically, this knowledge can be used by flanking a transgene with insulator elements generating a functionally independent domain protected from position effects at the insertion site of the transgene. Appropriate regulatory signaling can occur within the insulated-flanked domain as the insulators prevent accidental external chromatin interactions.

Factors like sequence-specific CTCF bound to insulators do not only organize the local chromatin interactions, but, in conjunction with other chromatin-associated elements like cohesins, are also involved in large-scale chromatin loop formation or coordination of topologically associated domains (TADs; see book $>$ Chap. 1 of Wutz)(Chen and Lei 2019; Pisignano et al. 2019). Hence, at many points in the genome the DNA sequence plays, through specific DNA-protein interactions, an important structuring role in organizing chromatin and, consequently, contribute to the epigenetic landscape. Since every cell of a defined organism contains the same DNA, the question is how this apparent rigid structure of chromatin, nevertheless, permits a highly dynamic environment for the regulation of basic nuclear activities in a cell-type specific manner. In the next section of this Chapter, we will discuss how nucleosome positioning is regulated.

\subsection{Nucleosome Remodeling}

\subsubsection{A Template for Transcription}

This nuclear process transcribes RNAs from genes that are located in different regions of the genomic templates. These transcription units range from ribosomal RNAs, tRNAs, and mRNAs to the newly discovered miRNAs and long non-coding RNAs that are involved in distinct biological processes. In all cases, the process of 
transcription is tightly controlled in a temporal and spatial manner. RNA polymerases have to pass through three phases: initiation, elongation, and termination (Cramer 2019). The initiation phase involves recognition of a promoter DNA sequence, opening of DNA strands, and synthesis of a short initial RNA oligomer (Cairns 2009). During the elongation phase, the polymerase uses the DNA template to synthesize the growing RNA chain in a processive manner. Finally, DNA and RNA are released during termination, and the polymerase can then be recycled and re-initiate transcription. This entire process is reflected in a characteristic arrangement of nucleosomes and accompanying histone modifications at the transcription unit. The promoter region is devoid of nucleosomes while the downstream region exerts regularly phased nucleosomes as a consequence of the repeated passage of the polymerase complex (• Fig. 2.1c, d).

Biochemically, the sequence of events can be ideally studied at an inducible gene, for example, a target gene activated by a nuclear hormone receptor (• Fig. 2.2). Under such conditions, the process can be started and analyzed in a synchronous manner by the addition of a hormone to a tissue culture. After hormone binding, the nuclear receptor dimerizes and enters the nucleus. With the help of accessory factors, it can bind to its DNA recognition site even in a nucleosomal context. To open chromatin structures, in a next step, remodeling factors are attracted (see book $>$ Chap. 1 of Wutz and below). The unwound DNA can be bound by general TFs and, consequently, the transcriptional preinitiation complex can be loaded onto the promoter.

In many instances the cofactor SAGA is a multifunctional complex that facilitates transcription initiation in the context of chromatin. The TFIID complex also acts as a cofactor that is involved in promoter recognition. Both SAGA and TFIID can deliver the TATA box-binding protein (TBP) to promoters and stimulate assembly of the preinitiation complex. Eukaryotic RNA polymerases require a number of general transcription factors for initiation. Assembly of the polymerase on promoters to form a pre-initiation complex, for example, requires the general transcription factors TFIIA, TFIIB, TFIID, TFIIE, TFIIF, and TFIIH. In the case of Pol II, TFs do not target the polymerase directly but, rather, through interactions with cofactors, like the multiprotein complex Mediator. After DNA opening, the DNA template strand transitions into the active center of the polymerase. The template strand then directs de novo RNA chain initiation without the use of a priming oligonucleotide. Extension of the RNA to a critical length eventually triggers disassembly of the preinitiation complex, escape of the polymerase from the promoter, and formation of a stable elongation complex.

The Pol II elongation complex often pauses downstream of the transcription start site (Gaertner and Zeitlinger 2014; Adelman and Lis 2012). Paused Pol II can be stabilized by the elongation factors DSIF and NELF but may also terminate (- Fig. 2.3). Upon an additional positive signal, Pol II is released from pausing into active elongation by the kinase positive transcription elongation factor, (P-TEFb). P-TEFb phosphorylates Pol II, DRB sensitivity-inducing factor (DSIF), and negative elongation factor (NELF), and this leads to dissociation of NELF and recruitment of elongation factors that stimulate Pol II progression. A number of epigenetic processes control transcription directly at the site of paused Pol II. Silencing by the Polycomb group (PcG) proteins (see book $>$ Chap. 3 of Paro) is the result of an inhibition of elongation by repressory PcG complexes. Transcriptional elongation is coupled to the processing of nascent RNA. This coupling requires the so-called 

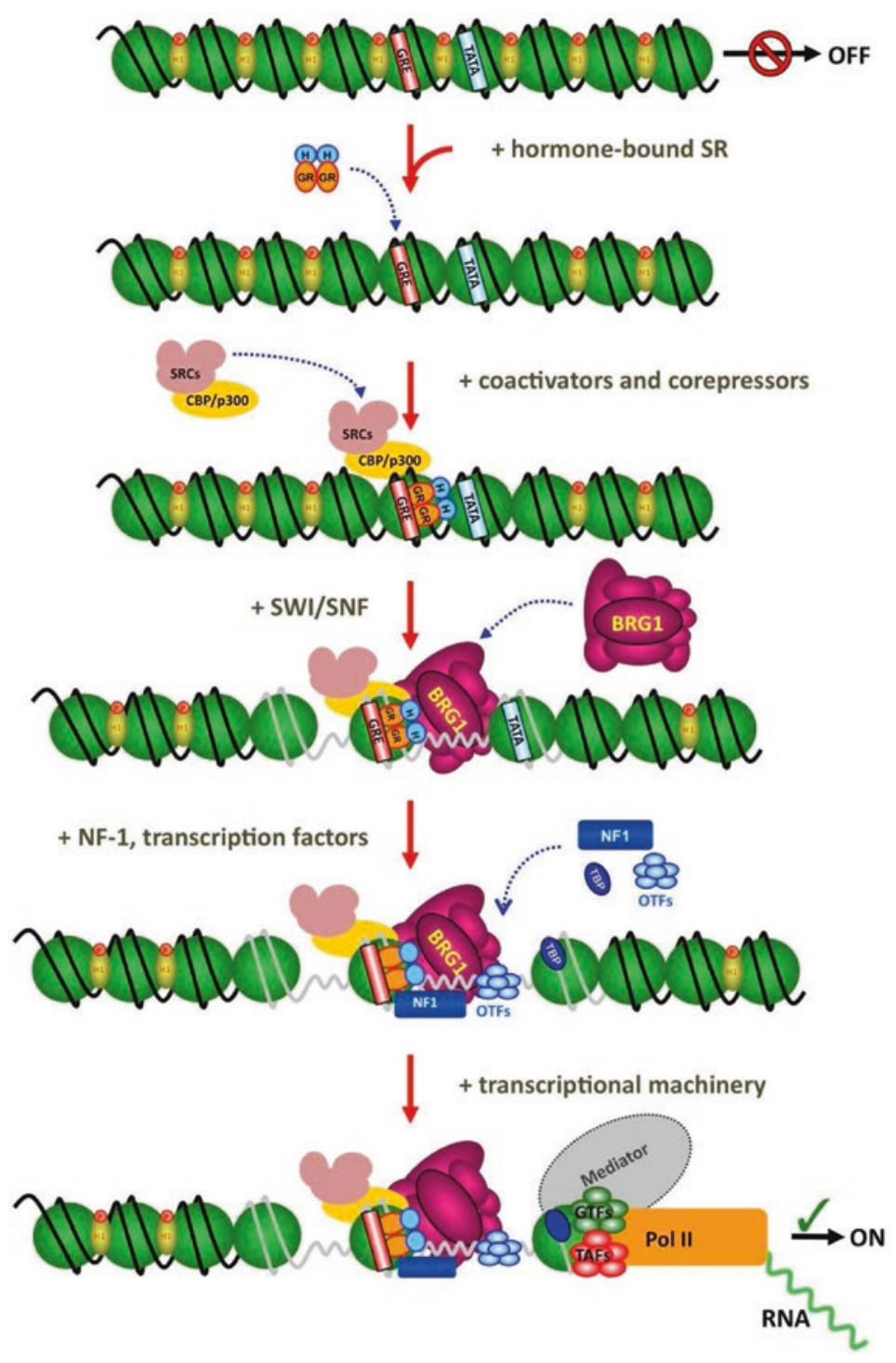

- Fig. 2.2 Key transition points during hormone signaling. After ligand binding, glucocorticoid receptor (GR) dimerizes, enters the nucleus, and binds target sequences such as the Glucocorticoid Response Element (GRE) within chromatin. The receptor binds co-regulators, such as NcoA1/SRC-1, NCoA2/TIF-2/GRP-1, and CBP/p300, which do not require remodeling to engage the receptor at the promoter. In the following step, these factors attract directly the BRG1 nucleosome remodeling complex SWI/SNF to the promoter leading to the formation of a nucleosome free region. This allows transcription factors such as NF1 and the octamer transcription factors (OTFs) as well as the TATA-binding protein (TBP) to engage with the promoter. Finally, mediator proteins are recruited and the preinitiation complex forms, leading to transcription by RNA Pol II. (From King et al. (2012)) 
a Promoter opening

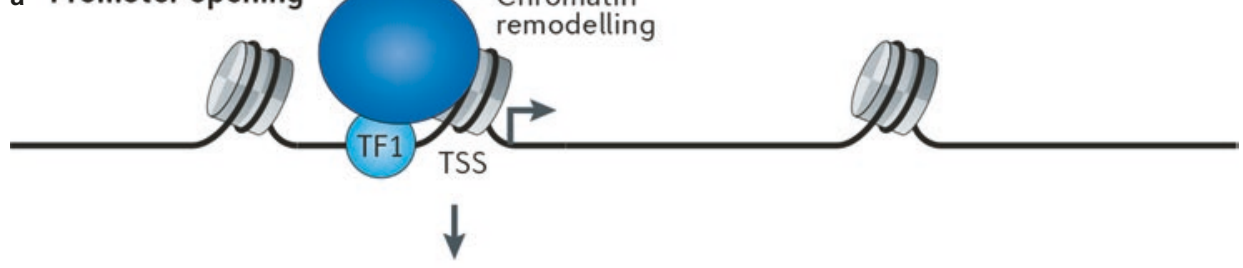

b Pre-initiation complex formation

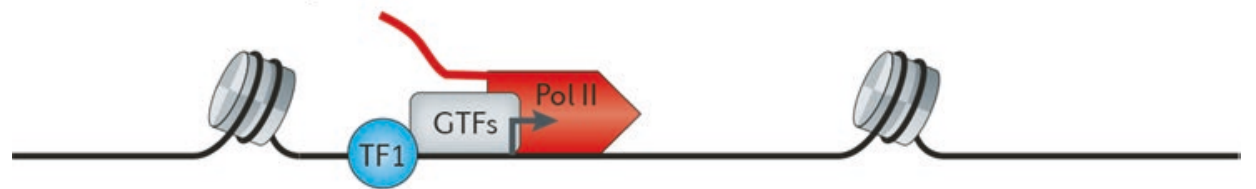

c Pausing
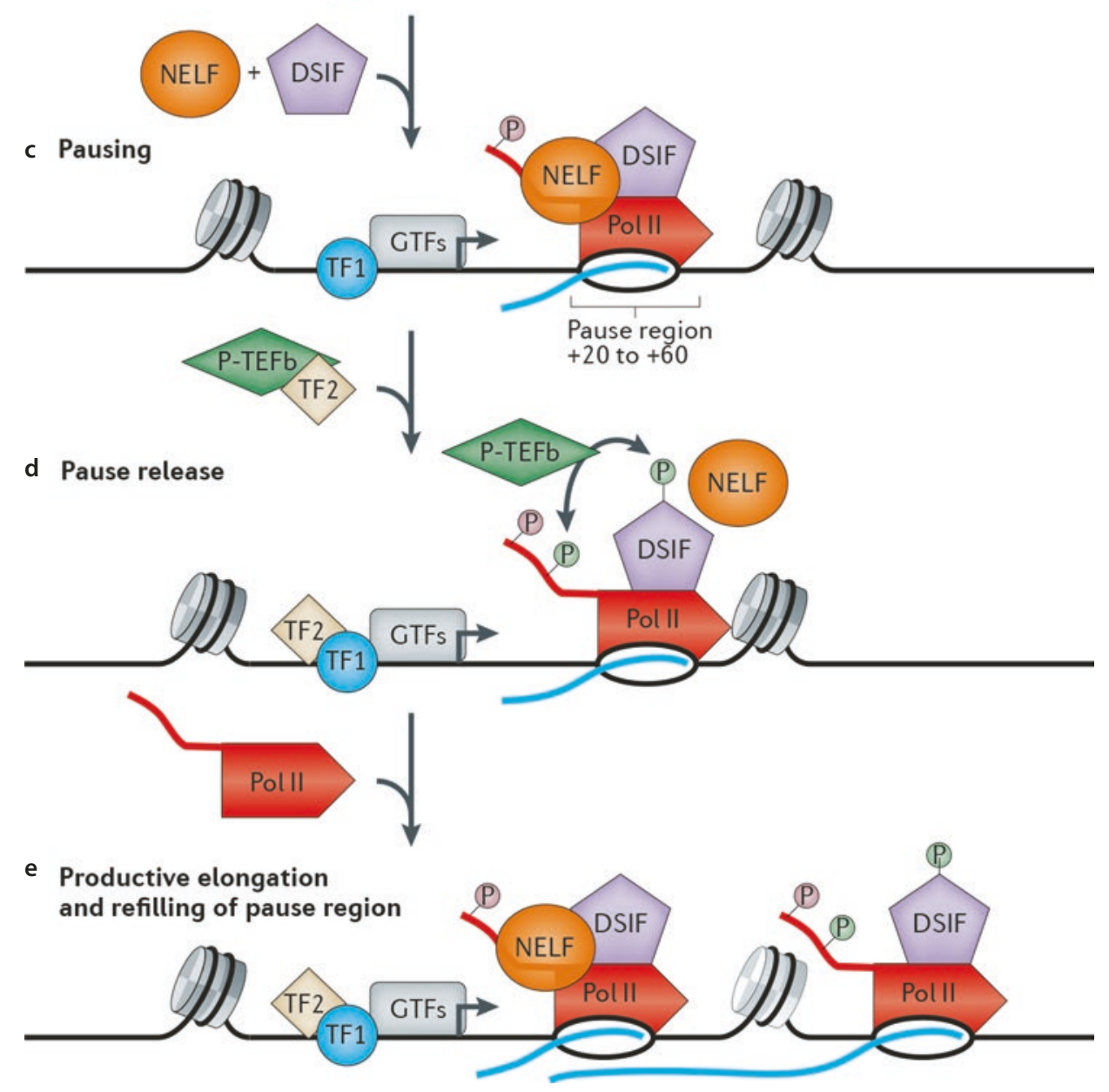
C-terminal repeat domain (CTD). During elongation, the CTD is highly phosphorylated and recruits factors for post-transcriptional processing, like pre-mRNA capping, splicing, and also enzymes that modify histones. Indeed, the chromatin landscape at a gene appears to have a profound impact on post-transcriptional events and influences for example the specificity of splicing events. At the end of the transcribed region, Pol II transcribes the poly-adenylation site, which results in cleavage of the nascent RNA transcript and maturation of the mRNA.

At every point of the transcription cycle, the factors described above interact with a chromatin template and, therefore, require additional activities of partners to make DNA more accessible. Pioneer TFs can recognize their cognate DNA binding sequence in a nucleosomal context and open up chromatin by attracting histone acetylase complexes like SAGA that locally destabilize repressive nucleosomal structures. However, to make DNA accessible over an extended region, as required for example for the assembly of the large transcriptional pre-initiation complex, an energy dependent machinery in the form of ATP-dependent chromatin remodeling complexes is necessary.

\subsubsection{Chromatin Remodeling Complexes}

The process of remodeling involves reconfiguring the histone-DNA interactions either by disrupting, reassembling, or moving nucleosomes (Sundaramoorthy 2019). Enzymes known as ATP-dependent chromatin remodeling complexes are capable of generating changes by binding to a single nucleosome and reconfiguring it through utilizing the energy obtained from hydrolyzing ATP. Remodelers can (i) mediate nucleosome sliding in which the nucleosome position on DNA moves in either direction, (ii) generate access for TFs whereby histones remain bound but DNA becomes

Fig. 2.3 Establishment and release of paused RNA polymerase II. The promoter region is shown with the transcription start site (TSS). Factors that are involved in the establishment or release of paused Pol II, such as DRB sensitivity-inducing factor (DSIF; purple pentagon), negative elongation factor (NELF; orange oval), and positive transcription elongation factor $\mathrm{b}$ ( $\mathrm{P}-\mathrm{TEFb}$; green diamond), are indicated. a Promoter opening often involves binding a sequence-specific transcription factor (shown here as TF1) that brings in chromatin remodelers to remove nucleosomes from around the TSS and to render the promoter accessible for recruitment of the transcription machinery. b Pre-initiation complex formation involves the recruitment of a set of general transcription factors (GTFs) and Pol II, which is also facilitated by binding specific transcription factors (also shown as TF1 for simplicity). This step precedes the initiation of RNA synthesis. c Pol II pausing occurs shortly after transcription initiation and involves the association of pausing factors DRB sensitivity-inducing factor (DSIF) and negative elongation factor (NELF). The paused Pol II is phosphorylated on its carboxy-terminal heptapeptide repeat domain (CTD). The region in which pausing takes place is indicated in the figure. $\mathbf{d}$ Pause release is triggered by the recruitment of the positive transcription elongation factor $\mathrm{b}(\mathrm{P}-\mathrm{TEFb}$ kinase), either directly or indirectly by a transcription factor (shown here as TF2). P-TEFb kinase phosphorylates the DSIF-NELF complex to release paused Pol II and also targets the CTD. Phosphorylation of DSIF-NELF dissociates NELF from the elongation complex and transforms DSIF into a positive elongation factor that associates with Pol II throughout the gene. e In the presence of both TF1 and TF2, escape of the paused Pol II into productive elongation is rapidly followed by entry of another Pol II into the pause site, allowing for efficient RNA production. When the gene is activated, some nucleosome disruption is likely, as depicted by the lighter coloring of the downstream nucleosome. (From Adelman and Lis (2012)) 


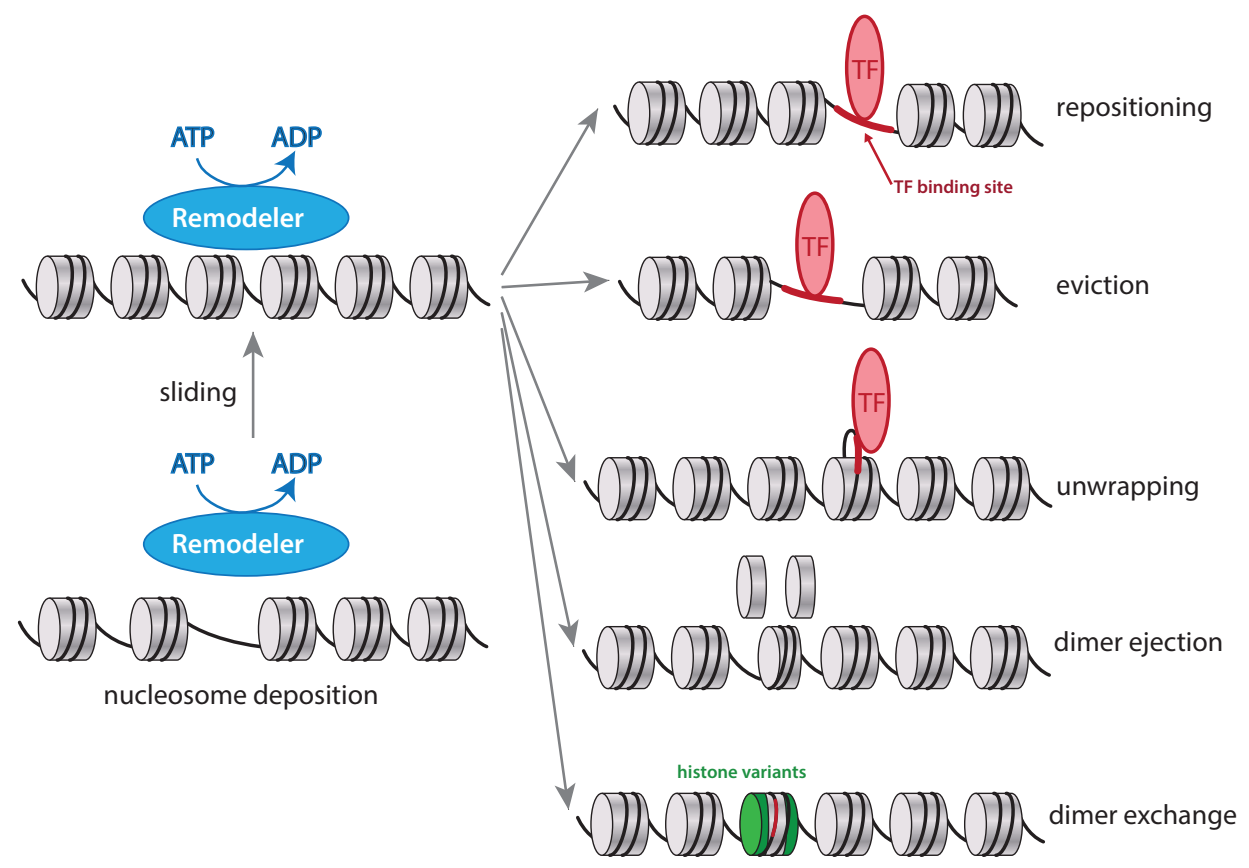

- Fig. 2.4 The activities of chromatin remodeling complexes. Remodelers support assembly of a regular chromatin template by sliding deposited nucleosome and thereby providing space for new nucleosomes. Remodelers can make a binding site for a TF accessible by moving nucleosomes to the side (repositioning), by ejecting a complete nucleosome (eviction) or by partially freeing the DNA (unwrapping). In addition, remodelers can change the local composition of nucleosomes by ejecting a dimer or replacing histone dimers with corresponding variant forms (dimer exchange)

more accessible, (iii) induce eviction of the histone octamer, and (iv) replace a core histone with a variant histone (• Fig. 2.4) (Lorch and Kornberg 2017; Clapier and Cairns 2009). The general importance of these processes is also evident by the fact that components of chromatin remodeling complexes are found to be frequently mutated in cancers (see book Chap. 8 of Santoro).

A shared property of this wide variety of effects rests on a common enzymatic function; an ATP-dependent DNA translocase activity that is utilized to break the histone-DNA contacts. This activity is carried out by an evolutionarily conserved catalytic core ATPase subunit. This remodeler ATPase subunit adopts a fold typical of the much larger superfamily 2 (SF2) DNA/RNA helicases and translocases. Analogous to SF2 family DNA translocases, the remodeler ATPase contains several highly conserved motifs, including WALKER A/B motifs, a DNA-binding cleft, and a site of ATP-binding that are all important for the DNA translocation activity. The ATPase of the remodeler differs from the SF2 DNA translocases in that they have much higher affinity for nucleosomes compared to naked DNA. An interesting aspect that emerged from the recently resolved CryoEM structures of various remodeler-nucleosome complexes is that remodelers unwrap outer turns of DNA from the nucleosome. This is a key step to minimize the number of histone-DNA contacts and maximize the output from ATP hydrolysis (• Fig. 2.5). Based on the shared domains/motifs within their ATPase subunit fine-tuning the remodeler 


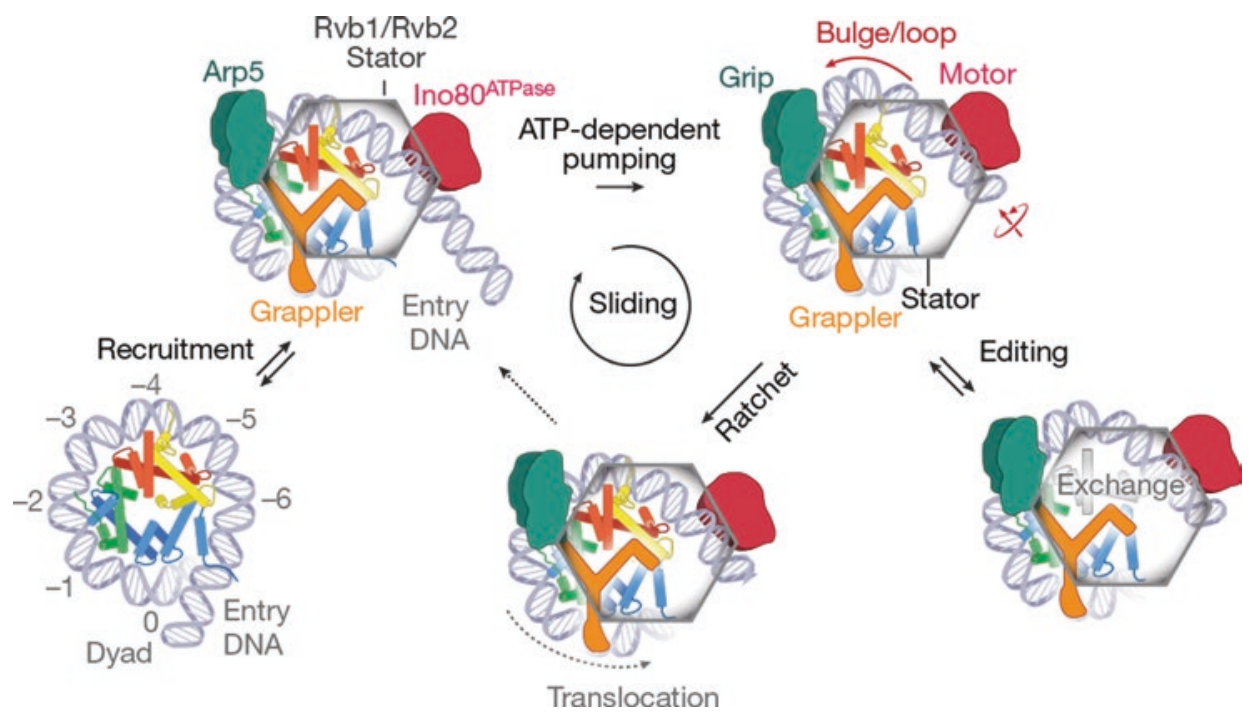

- Fig. 2.5 Model of INO80 nucleosome remodeling. The functional architecture of INO80 with motor (ATPase, red), grip (Arp complex, green) and grappler (orange) suggests that processive nucleosome sliding proceeds via a ratchet mechanism. Transient generation of loops between the motor and the grip could expose $\mathrm{H} 2 \mathrm{~A}-\mathrm{H} 2 \mathrm{~B}$ for editing. Direct binding of $\mathrm{H} 2 \mathrm{~A}-\mathrm{H} 2 \mathrm{~B}$ by the grappler sensorfoot could regulate variant- or modification-specific editing. (From Eustermann et al. (2018))

ATPase function and defining its selectivity for nucleosomal substrates, the family of Snf2 ATPases is grouped into four different classes: SWI/SNF, ISWI, CHD/Mi-2, and INO80-SWR1.

$S W I / S N F$ remodelers are regulators involved in a wide range of gene control processes in all cell types. Composed typically of 9-14 subunits, at least two forms of SWI/SNF-specific Snf2 ATPases are present in most cell types with well-conserved signature domains: an acetyl lysine-binding bromo domain, a helicase-related SANT domain that binds actin-related protein (ARP), and the SF2 family helicase domain. In Saccharomyces cerevisiae, these two forms are termed as SWI-SNF (contains ATPase Swi2, also called Snf2) and RSC (contains ATPase Sth1). SWI/SNF complexes can oppose epigenetic silencing by $\mathrm{PcG}$ proteins (see book $>$ Chap. 3 of Paro). In Drosophila melanogaster, $\mathrm{PcG}$ proteins were shown to maintain repression of Hox genes during embryogenesis, while the SWI/SNF complex promotes Hox gene activation. In mammalian cells, the SWI/SNF complexes are generally grouped into two types of complexes: hBAF complexes containing ATPase BRG1 or BRM and several alternate core subunits such as ARID1A or ARID1B and hPBAF (polybromo-associated BAF) complexes containing the BRG1 ATPase associated with factors such as PBRM1 and ARID2. Both are essential for cell survival and involved in a number of transcriptional processes.

The ISWI (imitation switch) remodelers were initially identified in Drosophila and then subsequently observed in other species like yeast, Xenopus, and human. In Drosophila, the complex exists in at least four forms: ACF, NURF, CHRAC, and RSF. All four of these complexes possess the same ATPase protein ISWI but contain different associated subunits, which are likely important for specialized remodeler functions. In yeast $S$. cerevisiae, there are two distinct ISWI complexes, ISW1 and 
ISW2. Mammals have two homologs of ISWI, SNF2L (encoded by the SMARCAI gene) and SNF2H (encoded by the SMARCA5 gene). In mammals, five ISWI complexes have been identified: ACF, CHRAC, WICH, RSF, and NoRC. ISWI complexes are typically heterodimers and composed of an ISWI protein $(\mathrm{SNF} 2 \mathrm{H}$ or SNF2L) and another subunit that provides specific biological processes. The ISWI remodelers generate regularly spaced nucleosomal arrays, facilitating the deposition of histones onto DNA and participating in replication progression and transcriptional repression.

The members of the chromodomain-helicase-DNA-binding domain (CHD) family are characterized by the presence of tandem chromodomains in the $\mathrm{N}$-terminal region of the ATPase and a C-terminal DNA-binding domain. This highly conserved family is divided into three subfamilies based on the presence of additional protein domains. The first subfamily is defined by an additional DNA-binding domain located in the C-terminal region. This subfamily consists of the $S$. cerevisiae Chd1, the only CHD family member in yeast, as well as Chd1 and Chd2 in higher eukaryotes. The second subfamily includes the proteins Chd 3 and Chd 4 as well as Drosophila Mi-2, which are characterized by the presence of an additional $\mathrm{N}$-terminal PHD domain. Members of the second subfamily are typically part of large multi-subunit complexes having besides chromatin remodeling functions, also histone deacetylation activities. The third subfamily contains the proteins Chd5Chd 9 and is characterized by additional functional motives in the C-terminal region, including paired BRK domains, a SANT-like domain, chromo domains, and a DNA-binding domain.

The remodelers of the INO80 family are the most recently identified remodeling complexes. The multi-subunit INO80 (Inositol 80) and SWR1 (Sick with Rat8) are the subfamily members of the INO80 family. INO80 regulates gene expression, DNA repair and replication by sliding nucleosomes, the exchange of histone H2A.Z with $\mathrm{H} 2 \mathrm{~A}$, and the positioning of +1 and -1 nucleosomes at promoter DNA. Recent cryo-EM studies resolved the interaction sites of INO80 with a nucleosome (see - Fig. 2.5) (Eustermann et al. 2018). Their analyses suggest a ratchet-like mechanism for how INO80 slides and possibly edits nucleosomes. INO80 unwraps and grips DNA and histones by multivalent interactions. The motor is positioned to pump DNA into the nucleosome against the Arp complex, which could hold onto DNA until a sufficient force is generated by multiple small steps of the motor. Such a tracking mechanism might create a DNA loop between the motor and the Arp complex. As a result, INO80 would move nucleosomes in larger steps of 10-20 bp. The proposed ratchet mechanism explains DNA loop formation that results in large translocation steps, as well as the means for ATP-dependent H2A.Z $\rightarrow$ H2A exchange. The motor, stator and multivalent grip of INO80 enable highly processive sliding without release and large-scale reconfigurations such as editing while keeping the remainder of the nucleosome intact.

Central to each of these remodeler families is the ATPase domain that conducts DNA translocase activity through ATP hydrolysis. Accessory domains within the ATPase and auxiliary subunits in a multi-subunit remodeler finetune the ATPase activity to the desired outcome of nucleosome remodeling. Hence, not only transcriptional processes require the moving capacity of the remodelers but all basic nuclear processes depend in one form or the other on this powerful competence to free DNA. 
Methods Box 2.1: Determining DNA Accessibility in a Chromatin Template As described in this chapter, the regulated access to the DNA template is a prerequisite for many nuclear enzymatic processes. In reverse, the appearance of accessible sites in chromatin could pinpoint to regions of regulation or active genomic processing. To this purpose, nuclei are treated with limited concentrations of nucleases like DNase I or micrococcal nuclease. Given the appropriate conditions, these enzymes will only cut exposed DNA but not DNA wrapped around histones or packaged in condensed or silenced nucleosomal structures like heterochromatin. Originally, open chromatin regions, termed DNase hypersensitive sites, could be determined at single genes. For example, after hormone induction, the chromatin rearrangements at the nuclear hormone binding sites and the accompanying changes at the promoter could be visualized by the appearance, over time, of DNase hypersensitive sites. Experimentally, after hormone induction of the cells in culture, the nuclei would be isolated and treated with nucleases under limiting conditions. The reaction would be stopped, the DNA isolated, its size further reduced with specific restriction enzymes, and eventually separated on an agarose gel. Hybridization with probes encompassing the boundaries of the gene would reveal the sites at which the nuclease attack had reduced the original size of the restriction fragment. Comparison of hypersensitive sites from uninduced and induced cells could provide not only information about the potential location of regulatory sites and produce co-called "DNase footprints" but also give clues about how fast chromatin is opening to permit the associated nuclear process (• Box Fig. 2.1).

In the meantime, recognition of hypersensitive sites can be achieved on a genomewide scale. While the initial steps of limited nuclease attack to open chromatin conformations remains principally the same, the subsequent site recognition utilizes new high-throughput sequencing technologies. After nuclease digestion, fragments of different sizes appear; while nucleosome-packaged DNA, in general, produces larger fragments, regions with open chromatin or nucleosome depleted regions are cut more often, resulting in smaller fragments. Hence, a local high frequency of smaller fragments is an indication for a hypersensitive site in the genome.

A further improvement was achieved with ATAC-Seq (Assay for TransposaseAccessible Chromatin using Sequencing). Instead of DNase I, a mutated form of a transposase, Tn5, is used to fragment the chromatin. This enzyme not only cleaves DNA but also tags the resulting double-stranded DNA fragment with sequencing adaptors, which can be amplified by PCR and subjected to high-throughput sequencing. Also in this case, the number of reads for a region correlates with how open that chromatin region is. The method has the advantage that much less starting material, compared to the other described methods, is required. Overall, the method needs less time as fewer steps are required and tricky enzyme titrations are not necessary.

These methods have provided substantial insight into how chromatin "breathes" under specific physiological conditions, presenting a direct readout for chromatin dynamics. In all cases, however, the approach is dependent on nucleases which have an inherent albeit low DNA sequence specificity. This could result in preferential site cleavage, which is not solely governed by the chromatin structure. These caveats have to be considered when generating and interpreting results measuring chromatin dynamics. 


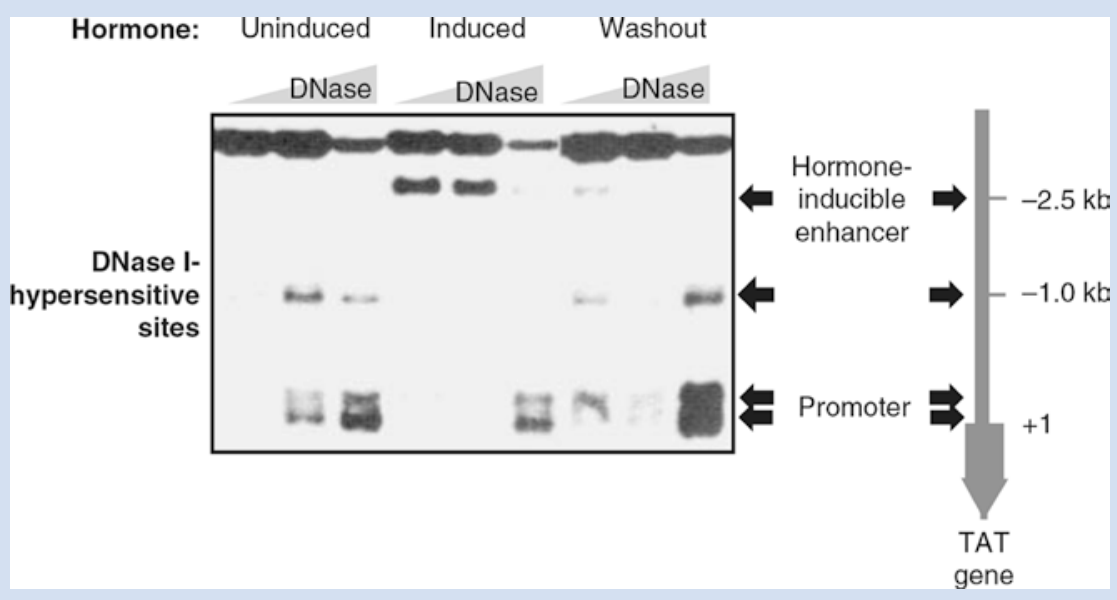

- Box Fig. 2.1 DNase I-hypersensitivity (DH) analysis at an inducible gene. The chromatin organization at the glucocorticoid-responsive enhancer element, $2.5 \mathrm{~kb}$ upstream of the promoter of the tyrosine aminotransferase gene, was probed in rat liver cells. Isolated nuclei of cells are digested with increasing amounts of DNase I. Digested genomic DNA is purified, cleaved with a restriction enzyme, resolved by agarose gel electrophoresis, and subjected to Southern blotting. The DH sites are revealed by indirect end-labeling of restriction fragments through hybridization of a small radioactive probe (marked with arrows). In the uninduced state there are two DH sites at the promoter and one at $21 \mathrm{~kb}$ upstream. After hormone induction with corticosterone, nucleosomes are remodeled at the enhancer within $15 \mathrm{~min}$. A new DH site appears $2.5 \mathrm{~kb}$ upstream of the transcriptional start site. This correlates with the binding of glucocorticoid receptors and a complex set of remodeling factors. On removal of the hormone ("washout"), the factors dissociate and canonical nucleosomes reform within 15 min and the 22.5-kb enhancer DH disappears. (From Becker and Workman (2013))

\subsection{Nucleosome Assembly}

The above section discussed processes evicting, moving, or replacing histones in nucleosomes and, thereby, liberating DNA for access to processing enzymes. However, the reverse process is as important in order to reconstitute the native nucleosomal template and the correct epigenetic landscape. This need is most evident at the time of DNA replication. The newly synthesized DNA has to be packaged and epigenetically marked in a regular and complementary manner. At the replication fork, a highly orchestrated mechanism not only templates and produces an identical copy of the DNA but also removes nucleosomes in front and reassembles histones into nucleosomes behind, now on two DNA fibers in an epigenetically impeccable state (Hammond et al. 2017).

\subsubsection{Histone Variants and Histone Chaperones}

Most histones are produced during the S-phase of the cell cycle. These DNA synthesis-coupled canonical histones provide the source for the repackaging of the two new chromatids. However, a number of DNA synthesis-independent histone 
variants are produced at any time in the cell cycle, with variable tasks in structuring the chromatin landscape (see also book $>$ Chap. 1 of Wutz) (Talbert and Henikoff 2017). The incorporation of the centromeric H3 variant (known as CENPA in vertebrates or CENH3 in plants) instead of the canonical H3 into a nucleosome forms the foundational structure of centromeric chromatin. The replacement of human canonical H3.1 by the highly similar variant $\mathrm{H} 3.3$ has few apparent consequences. This variant has a primary role in repairing gaps in the chromatin landscape that result from nucleosome disruption occurring during basic nuclear processes. Several H2A variants affect gene expression: $\mathrm{H} 2 \mathrm{~A} . \mathrm{Z}$ and $\mathrm{H} 2 \mathrm{~A} . \mathrm{B}$ are implicated in transcription initiation whereas macroH2A in animals and $\mathrm{H} 2 \mathrm{~A}$.W in plants seem to be associated with nucleosome immobility and transcriptional silencing. Hence, like DNA containing base variants like methyl-cytosine, also nucleosomes can alter their information surface by incorporating variants of the canonical histones. This provides an additional layer of flexibility and complexity for epigenetic processing as the variants can have an influence on nucleosome stability and marking by chromatin modifiers.

For the assembly of histones into nucleosomes histone chaperones are required (De Koning et al. 2007). Indeed, when histones are mixed with DNA in solution at physiological ionic strength, they precipitate unless chaperones help histones to fold properly, preventing their positive charges from engaging in nonspecific interactions. The highly conserved histone chaperones are also necessary for escorting and depositing histones into chromatin and for histone storage. There are a number of different chaperones for cellular processes and pathways of histone assembly. Newly synthesized histones are bound by a series of chaperones in the cytosol before entering the nucleus. Once soluble histone complexes enter the nucleus, chromatin assembly factor 1 (CAF1) directs histone assembly behind the replication fork, whereas the histone regulator A (HIRA) complex directs assembly into chromatin independent of DNA synthesis (see below). In humans, CAF1 deposits canonical H3.1 and H3.2 during replication, whereas HIRA deposits the H3.3 variant throughout the cell cycle. Hence, any process in the cell requiring histone assembly utilizes the chaperoning capability of specific complexes. - Figure 2.6 summarizes the identity and sites of action of these important factors in chromatin dynamics.

\subsubsection{The Replication Fork: Still the Major Enigma in Epigenetics}

The most outstanding cooperation between DNA, histones, histone chaperones, chromatin modifiers, and the replication machinery is exemplified at the replication fork (- Fig. 2.7) (Bellush and Whitehouse 2017; Hammond et al. 2017). The passage of genomic DNA through the small hole in the replicative helicase must result in the transient release of all DNA-binding proteins, including the disassembly of nucleosomes and, hence, the uncoupling of histone- and nucleosome-based epigenetic information from the DNA. Eventually, old nucleosomes, including many with histone variants and modifications, are rapidly reassembled on the newly synthesized leading and lagging strands. Gaps between old nucleosomes are filled with nucleosomes comprising newly synthesized canonical histones. Histone chaperones support this dynamic process. Behind the replication fork deposition of new nucleosomes is mediated by the histone chaperone CAF1, which teams with the replication clamp proliferating cell nuclear antigen (PCNA) and travels with the replisome. CAF1 is a trimeric complex 


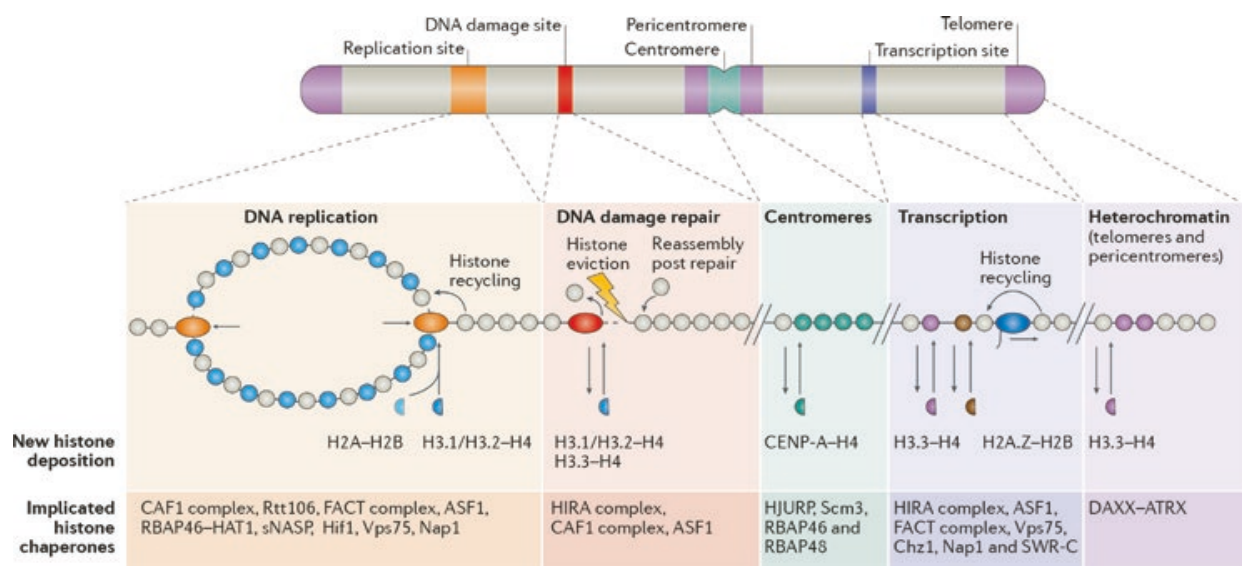

Fig. 2.6 Overview of histone deposition mechanisms. Newly synthesized histones are incorporated into chromatin via globally and locally acting mechanisms. A network of specialized histone chaperones controls histone delivery and deposition. The figure provides an overview of replication-coupled and replication-independent pathways that require the incorporation of newly synthesized canonical histones and replacement variants, together with parental histone recycling. The histone chaperones that are implicated in each process are listed at the bottom. (From (Hammond et al. (2017))

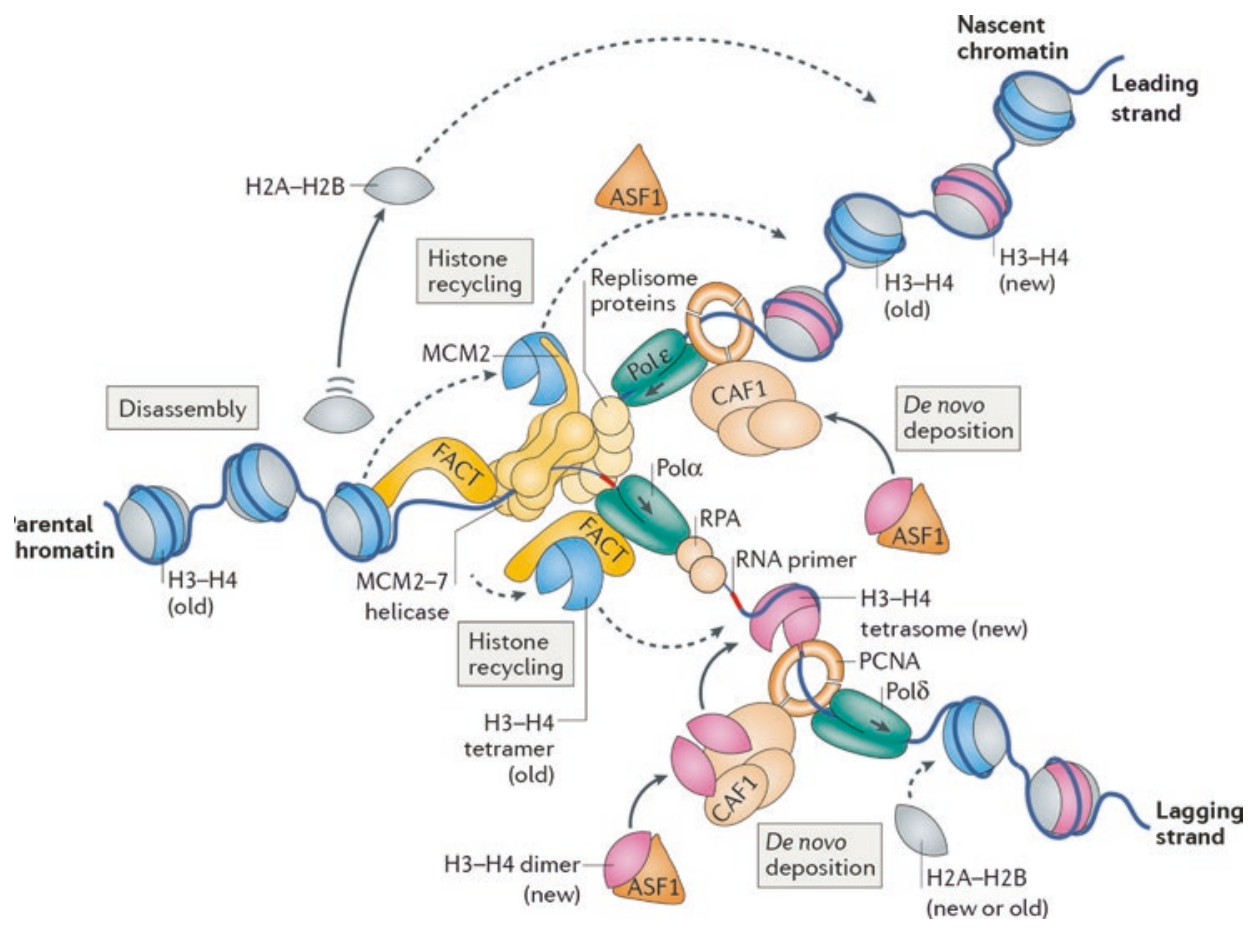

- Fig. 2.7 Parental histone recycling during DNA replication. Evicted parental histones are randomly segregated to daughter DNA strands. It remains unknown whether other components of the DNA replication machinery have histone chaperone activity and whether deposition of old and new histones occurs by separate pathways (for details see text). (From Hammond et al. (2017)) 
responsible for assembling new canonical H3 nucleosomes behind the fork (see - Fig. 2.7). ASF1 interacts with H3-H4 by binding the homodimerization interface of $\mathrm{H} 3$ and can only present dimers, not tetramers, to CAF1. A single CAF1 molecule can bind two H3-H4 dimers or a crosslinked (H3-H4), tetramer, suggesting that CAF1 is capable of assembling and depositing (H3-H4) tetramers onto DNA. Hence, old nucleosomes might be randomly disassembled and reassembled from their dimer components behind the fork, resulting in histone octamers containing both new and old histones. However, new Histones $\mathrm{H} 3$ and $\mathrm{H} 4$ may have epigenetic marks added before chromatin assembly at the fork. It is thought that $\mathrm{H} 3 \mathrm{~K} 9$ monomethylation and $\mathrm{H} 4 \mathrm{~K} 5$ and H4K12 acetylation are commonly added moieties by modifying enzymes SetDB1 and HAT1, respectively, in complex with histone chaperones (e.g., CAF1 or ASF1). Once chromatin has been assembled these acetylation marks might become removed by deacetylases and replaced by corresponding opposing marks like $\mathrm{H} 3 \mathrm{~K} 9 \mathrm{me} 3$ in the case of heterochromatin. However, in animals, canonical H3.1-containing nucleosomes consist of nearly all old or all new (H3.1-H4) tetramers. This suggests that either old tetramers are transferred intact behind the fork or they are transiently disassembled and reassembled in a way that excludes the incorporation of new dimers. Transfer of tetramers or dimers occurs across the fork either to CAF1 via ASF1 or, perhaps with the assistance of FACT (Facilitates Chromatin Transcription), directly onto DNA. FACT may contribute to nucleosome disruption and histone recycling through interaction with the replicative helicase CMG complex (which includes CDC45-MCM2-7-GINS), polymerase $\alpha$ (Pol $\alpha$ ), and the single-strand binding replication protein A (RPA). MCM2 provides a binding platform for evicted $\mathrm{H} 3-\mathrm{H} 4$ tetramers and may facilitate their recycling directly, in collaboration with FACT, or as dimers with ASF1, which splits H3-H4 tetramers and forms a co-chaperone complex with MCM2.

The big question still is how, after the passage of the replication fork and the assembly of new and old nucleosomes, the local epigenetic landscape is reestablished (Singh 2014). A templating mechanism has to duplicate and appropriately pattern the new histones with the necessary modifications. Given the vicinity to the parental histones, these could act as anchors and templates for readers, writers and erasers to appropriately tag the new histones (see book $>$ Chap. 1 of Wutz). In light of the intricate patterns of modifications observed at certain functional chromatin locations, this certainly begs the question which and how precisely a biochemical mechanism is capable to faithfully reproduce these instructions. A better understanding of this process represents certainly a major and ambitious challenge in epigenetic research.

\section{Take-Home Message}

- The assembly of histones into nucleosomes is influenced by the DNA sequences wrapped around the protein core. Certain sequences favor bending of DNA whereas others disfavor the assembly of a nucleosome. Hence, the chromatin landscape is to a large part also determined by the genome sequence.

- DNA elements organize the chromatin in the 3D space of the nucleus. Insulator/ boundary elements prevent long-range effects of repressive heterochromatin but can also locally control the interaction of cis-regulatory elements. 
- DNA-templated processes like transcription are initiated by a well-controlled cascade of chromatin remodeling. Pioneer TFs provide sequence recognition without being inhibited by the nucleosomal structure and locally attract histone modifiers generating activating marks. ATP-driven chromatin remodeling complexes produce an extended local disruption of nucleosomes and partial replacement of canonical histones with variants. Correspondingly, open chromatin can be processed by polymerases to synthesize the required products.

- Either after DNA replication or after execution of other basic nuclear processes, the chromatin structure has to be fully reestablished. This does not only require the assembly of histones into a nucleosomal structure but also the maintenance of the appropriate epigenetic landscape defined by histone modifications, DNA methylation patterns, and others. The transport, storage, and local assembly of histones into nucleosomes is fostered by histone chaperones. In cooperation with the factors governing the basic nuclear processes and epigenetic processing, they ensure that the chromatin template is faithfully restored.

\section{References}

Adelman K, Lis JT (2012) Promoter-proximal pausing of RNA polymerase II: emerging roles in metazoans. Nat Rev Genet 13(10):720-731. https://doi.org/10.1038/nrg3293

Becker PB, Workman JL (2013) Nucleosome remodeling and epigenetics. Cold Spring Harb Perspect Biol 5(9). https://doi.org/10.1101/cshperspect.a017905

Bellush JM, Whitehouse I (2017) DNA replication through a chromatin environment. Philos Trans R Soc Lond Ser B Biol Sci 372(1731). https://doi.org/10.1098/rstb.2016.0287

Cairns BR (2009) The logic of chromatin architecture and remodelling at promoters. Nature 461(7261):193-198. https://doi.org/10.1038/nature08450

Chen D, Lei EP (2019) Function and regulation of chromatin insulators in dynamic genome organization. Curr Opin Cell Biol 58:61-68. https://doi.org/10.1016/j.ceb.2019.02.001

Clapier CR, Cairns BR (2009) The biology of chromatin remodeling complexes. Annu Rev Biochem 78:273-304. https://doi.org/10.1146/annurev.biochem.77.062706.153223

Cramer P (2019) Eukaryotic transcription turns 50. Cell 179(4):808-812. https://doi.org/10.1016/j. cell.2019.09.018

De Koning L, Corpet A, Haber JE, Almouzni G (2007) Histone chaperones: an escort network regulating histone traffic. Nat Struct Mol Biol 14(11):997-1007. https://doi.org/10.1038/nsmb1318

Eustermann S, Schall K, Kostrewa D, Lakomek K, Strauss M, Moldt M, Hopfner KP (2018) Structural basis for ATP-dependent chromatin remodelling by the INO80 complex. Nature 556(7701):386390. https://doi.org/10.1038/s41586-018-0029-y

Gaertner B, Zeitlinger J (2014) RNA polymerase II pausing during development. Development 141(6):1179-1183. https://doi.org/10.1242/dev.088492

Hammond CM, Stromme CB, Huang H, Patel DJ, Groth A (2017) Histone chaperone networks shaping chromatin function. Nat Rev Mol Cell Biol 18(3):141-158. https://doi.org/10.1038/nrm.2016.159

Hughes AL, Rando OJ (2014) Mechanisms underlying nucleosome positioning in vivo. Annu Rev Biophys 43:41-63. https://doi.org/10.1146/annurev-biophys-051013-023114

King HA, Trotter KW, Archer TK (2012) Chromatin remodeling during glucocorticoid receptor regulated transactivation. Biochim Biophys Acta 1819(7):716-726. https://doi.org/10.1016/j. bbagrm.2012.02.019

Lorch Y, Kornberg RD (2017) Chromatin-remodeling for transcription. Q Rev Biophys 50:e5. https:// doi.org/10.1017/S003358351700004X 
Misteli T, Soutoglou E (2009) The emerging role of nuclear architecture in DNA repair and genome maintenance. Nat Rev Mol Cell Biol 10(4):243-254. https://doi.org/10.1038/nrm2651

Papamichos-Chronakis M, Peterson CL (2013) Chromatin and the genome integrity network. Nat Rev Genet 14(1):62-75. https://doi.org/10.1038/nrg3345

Parmar JJ, Padinhateeri R (2020) Nucleosome positioning and chromatin organization. Curr Opin Struct Biol 64:111-118. https://doi.org/10.1016/j.sbi.2020.06.021

Pisignano G, Pavlaki I, Murrell A (2019) Being in a loop: how long non-coding RNAs organise genome architecture. Essays Biochem 63(1):177-186. https://doi.org/10.1042/EBC20180057

Rohs R, Jin X, West SM, Joshi R, Honig B, Mann RS (2010) Origins of specificity in protein-DNA recognition. Annu Rev Biochem 79:233-269. https://doi.org/10.1146/annurev-biochem-060408-091030

Singh J (2014) Role of DNA replication in establishment and propagation of epigenetic states of chromatin. Semin Cell Dev Biol 30:131-143. https://doi.org/10.1016/j.semcdb.2014.04.015

Struhl K, Segal E (2013) Determinants of nucleosome positioning. Nat Struct Mol Biol 20:267-273

Sundaramoorthy R (2019) Nucleosome remodelling: structural insights into ATP-dependent remodelling enzymes. Essays Biochem 63(1):45-58. https://doi.org/10.1042/EBC20180059

Talbert PB, Henikoff S (2017) Histone variants on the move: substrates for chromatin dynamics. Nat Rev Mol Cell Biol 18(2):115-126. https://doi.org/10.1038/nrm.2016.148

Valenzuela L, Kamakaka RT (2006) Chromatin insulators. Annu Rev Genet 40:107-138. https://doi. org/10.1146/annurev.genet.39.073003.113546

Open Access This chapter is licensed under the terms of the Creative Commons Attribution 4.0 International License (http://creativecommons.org/licenses/by/4.0/), which permits use, sharing, adaptation, distribution and reproduction in any medium or format, as long as you give appropriate credit to the original author(s) and the source, provide a link to the Creative Commons license and indicate if changes were made.

The images or other third party material in this chapter are included in the chapter's Creative Commons license, unless indicated otherwise in a credit line to the material. If material is not included in the chapter's Creative Commons license and your intended use is not permitted by statutory regulation or exceeds the permitted use, you will need to obtain permission directly from the copyright holder.

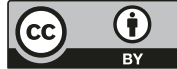

